\title{
Robust, small-scale cultivation platform for Streptomyces coelicolor
}

Sujata Vijay Sohoni ${ }^{1,2+}$, Prashant Madhusudan Bapat ${ }^{1,3+}$ and Anna Eliasson Lantz ${ }^{1 *}$

\begin{abstract}
Background: For fermentation process and strain improvement, where one wants to screen a large number of conditions and strains, robust and scalable high-throughput cultivation systems are crucial. Often, the time lag between bench-scale cultivations to production largely depends on approximate estimation of scalable physiological traits. Microtiter plate (MTP) based screening platforms have lately become an attractive alternative to shake flasks mainly because of the ease of automation. However, there are very few reports on applications for filamentous organisms; as well as efforts towards systematic validation of physiological behavior compared to larger scale are sparse. Moreover, available small-scale screening approaches are typically constrained by evaluating only an end point snapshot of phenotypes.

Results: To address these issues, we devised a robust, small-scale cultivation platform in the form of MTPs (24square deepwell) for the filamentous bacterium Streptomyces coelicolor and compared its performance to that of shake flasks and bench-scale reactors. We observed that re-designing of medium and inoculum preparation recipes resulted in improved reproducibility. Process turnaround time was significantly reduced due to the reduction in number of unit operations from inoculum to cultivation. The incorporation of glass beads ( $\varnothing 3 \mathrm{~mm}$ ) in MTPs not only improved the process performance in terms of improved oxygen transfer improving secondary metabolite production, but also helped to transform morphology from pellet to disperse, resulting in enhanced reproducibility. Addition of MOPS into the medium resulted in $\mathrm{pH}$ maintenance above 6.50, a crucial parameter towards reproducibility. Moreover, the entire trajectory of the process was analyzed for compatibility with bench-scale reactors. The MTP cultivations were found to behave similar to bench-scale in terms of growth rate, productivity and substrate uptake rate and so was the onset of antibiotic synthesis. Shake flask cultivations however, showed discrepancy with respect to morphology and had considerably reduced volumetric production rates of antibiotics.

Conclusion: We observed good agreement of the physiological data obtained in the developed MTP platform with bench-scale. Hence, the described MTP-based screening platform has a high potential for investigation of secondary metabolite biosynthesis in Streptomycetes and other filamentous bacteria and the use may significantly reduce the workload and costs.
\end{abstract}

\section{Background}

Streptomycetes produce numerous primary as well as secondary metabolites. Currently more than half of the antibiotics in clinical use are produced by Streptomyces species [1,2]. Traditionally Streptomyces strain improvement programs have been dominated by random mutagenesis, followed by screening and selection in

\footnotetext{
* Correspondence: ael@bio.dtu.dk

+ Contributed equally

${ }^{1}$ Center for Microbial Biotechnology, Department of Systems Biology, Technical University of Denmark, Building 223, DK-2800 Kgs Lyngby, Denmark

Full list of author information is available at the end of the article
}

controlled environments for a desired phenotype [3]. Today, with the advent of industrial systems biology [4], this traditional approach is complemented with targeted genetic strategies to increase yield, titer, productivity, and/or robustness [5,6]. Similarly, medium optimization strategies have also played a significant role in improving the productivity in antibiotic cultivation processes. The fate of these high-throughput approaches in capturing the desired phenotype lies in the successful scaled down [7] version of the production process i.e. to keep the physiological traits constant when the scale is changed. Moreover, such low volume, high-throughput 
cultivation platforms (HTPs) can also be used in other process optimization projects [8]. Small scale optimization of bioprocesses using microtiter plates (MTPs) has generated considerable interest in the research community over the last years [9-14]. However, efforts towards low volume cultivations for filamentous organism have been sparse [15,16]. Major challenges in development of a HTP for filamentous organisms are attributed to (i) unit operations involved from spore plate to cultivation, which make the process low-throughput as well as add variability and ii) morphology, that adds noise in the cultivation process resulting in lower reproducibility, thereby a less robust performance. Furthermore, $\mathrm{pH}$ management might be difficult in MTPs, however, this is a general issue not only valid for filamentous organisms. The efforts toward validating MTP in terms of scale comparison (bench-scale reactor versus MTP) and reproducibility at trajectory scale are sparse. Hence, MTP is typically applied as an end point snapshot tool for screening, whereas less attention has been paid towards mapping the entire trajectory of the underlying phenotype. This could be critical as microorganisms may have more than one optimum for deriving the same phenotype [17].

In this communication we report for the first time a systematic scale down approach for $S$. coelicolor cultivations that includes evaluation of kinetic physiological parameters and not only end point values. An optimization of the MTP cultivation was undertaken aiming at reducing process time as well as to enhance reproducibility and obtain a performance similar to that of benchscale reactors. The developed platform was validated for reproducibility and parameters were compared with the ones obtained from the bioreactor cultivations.

\section{Materials and Methods}

Solvents were HPLC grade and all other chemicals were analytical grade and purchased from Sigma -Aldrich (Steinheim, Germany) unless otherwise stated. Water (MQ) was purified using a Milli-Q-system (Millipore, Bradford, MA).

24-square deepwell microtiter plates and sandwich covers that contained special filters to minimize water evaporation during cultivations were obtained from Enzyscreen BV (Leiden, Netherlands) and used for all MTP cultivations in this study.

\section{Strain}

Streptomyces coelicolor A3(2) was a kind gift from Mervyn Bibb, John Innes Centre, Norwich, UK. The cultures of S. coelicolor were cultivated from frozen mycelia (FM) vials as described below. Preparation of frozen mycelia The protocol described by Borodina et al. (2008) for inoculum preparation of $S$. coelicolor was modified as shown in Figure 1. S. coelicolor was grown on Soya flour mannitol agar plates until well sporulated. Spores were harvested with $2 \mathrm{ml}$ of $20 \%$ (v/v) glycerol and filtered through glass fiber wool to remove mycelial fragments. The resulting spore suspension was then used to inoculate two $500 \mathrm{ml}$ volume baffled shake flasks with $50 \mathrm{ml}$ 2X YT medium and 30-40 glass beads (3 mm diameter) as described by Borodina et al. (2008). The shake flasks (SF) were incubated at $28^{\circ} \mathrm{C}$ and $150 \mathrm{rpm}$ [18]. One of the flasks was used to follow the growth ( $\mathrm{pH}$ and optical density measurements) by taking duplicate samples every $3 \mathrm{hrs}$. The other flask, to be used for frozen mycelia (FM) preparation, was harvested when the culture had reached mid exponential phase, typically at $\mathrm{pH}$ 8.1. The entire broth was centrifuged at $4,000 \times$ g for 10 min at $4^{\circ} \mathrm{C}$; care was taken not to include the beads while transferring the broth to a sterile falcon tube. The supernatant was discarded aseptically and the pellet was resuspended in $3 \mathrm{ml}$ of $20 \%(\mathrm{w} / \mathrm{v})$ precooled sterile peptone. The falcon tube was kept on ice for five minutes to prevent rise in the temperature during crushing. The mixture was then transferred into a pestle and tube glass homogenizer (in-house fabricated) and crushed gently moving the pestle up and down five times. $7 \mathrm{ml}$ peptone was added to the crushed mycelia. $\mathrm{OD}_{450 \mathrm{~nm}}$ of the resulting mycelia stock was measured and recorded $\left(\mathrm{OD}_{450 \mathrm{~nm}}\right.$ at around 25$)$. The mycelia stock solution was dispensed into cryo vials and stored at $-20^{\circ} \mathrm{C}$.

The purity of FM stock was checked by inoculation in nutrient broth and plating on nutrient agar plates. All the steps described above were carried out in laminar airflow to ensure aseptic conditions.

\section{Preparation of medium}

The defined minimal medium used for bench-scale cultivations was a modification to Evans medium (1970), and was limited in phosphate. The medium was prepared as described by Borodina et al. (2008) and contained $3 \mathrm{mM} \mathrm{NaH}_{2} \mathrm{PO}_{4}, 100 \mathrm{mM} \mathrm{NH}_{4} \mathrm{Cl}, 10 \mathrm{mM} \mathrm{KCl}, 2$ $\mathrm{mM} \mathrm{Na}_{2} \mathrm{SO} 4,2 \mathrm{mM}$ citric acid as chelating agent, 1.25 $\mathrm{mM} \mathrm{MgCl} 2,0.25 \mathrm{mM} \mathrm{CaCl}_{2}$, as well as the following per liter: 30 g glucose, $5 \mathrm{ml}$ trace elements solution (20 $\mathrm{mM} \mathrm{FeCl} 3,10 \mathrm{mM} \mathrm{CuCl}$, $50 \mathrm{mM} \mathrm{ZnCl}, 10 \mathrm{mM}$ $\mathrm{MnCl}_{2}, 0.02 \mathrm{mM} \mathrm{Na}_{2} \mathrm{MoO}_{4}, 20 \mathrm{mM} \mathrm{CoCl} 2,10 \mathrm{mM}$ $\left.\mathrm{H}_{3} \mathrm{BO}_{4}\right), 1 \mathrm{ml}$ vitamins solution $(0.05 \mathrm{~g}$ of biotin, $1 \mathrm{~g}$ of calcium pantothenate, $1 \mathrm{~g}$ of nicotinic acid, $25 \mathrm{~g}$ of myo-inositol, $1 \mathrm{~g}$ of thiamine- $\mathrm{HCl}, 1 \mathrm{~g}$ of pyridoxine$\mathrm{HCl}, 0.2 \mathrm{~g}$ of para-aminobenzoic acid/liter) and $100 \mu \mathrm{l}$ of organic antifoam. The medium used for MTPs and shake flasks was exactly the same as the one for benchscale cultivations, except that it contained 3-(N-morpholino) propanesulfonic acid (MOPS) as buffer and no antifoam. It should be noted that for un-optimized MTPs, $100 \mathrm{mM}$ 2-(N-morpholino) ethanesulfonic acid 


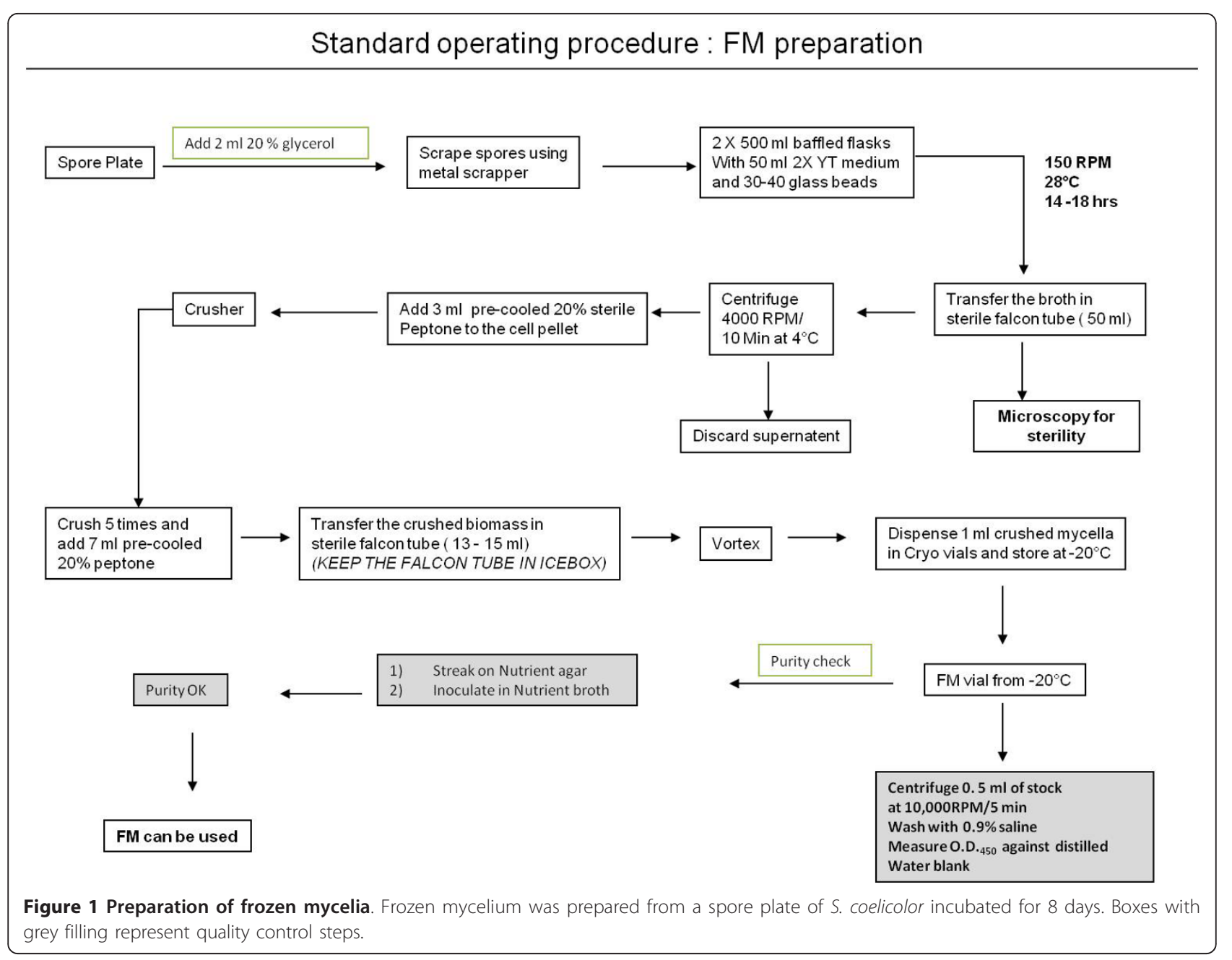

(MES) was used as a buffer instead of MOPS. Medium $\mathrm{pH}$ for MTPs and shake flasks was adjusted in the range of 6.80 to 6.90 using sterile $\mathrm{NaOH}$ solution $(4 \mathrm{M})$.

\section{Sterilization of bioreactors, shake flasks and MTPs}

Bioreactors were sterilized with the medium at $121^{\circ} \mathrm{C}$ for 40 minutes. Sterile glucose and vitamins were aseptically added to the sterile bioreactor.

Baffled shake flasks used for cultivations were autoclaved with $30-403 \mathrm{~mm}$ glass beads at $121^{\circ} \mathrm{C}$ for 40 minutes. The flasks were retained at $55^{\circ} \mathrm{C}$ for removal of moisture if any.

Each MTP well was filled with glass beads. The entire assembly was placed in a sterilization pouch (Westfield medicals, UK), heat sealed and autoclaved at $121^{\circ} \mathrm{C}$ for 35 to 40 minutes. All the MTPs were kept in wrapped condition at $55^{\circ} \mathrm{C}$ before inoculation to remove moisture if any.

For shake flask and MTP cultivations, the medium was autoclaved separately for 20 minutes.

\section{Cultivation conditions}

Batch cultivations were performed in bioreactors with 1 L working volume (Applikon Biotechnology, Schiedam, Netherlands) at $28^{\circ} \mathrm{C}, \mathrm{pH} 6.80-6.90,500 \mathrm{rpm}$ agitation rate and a $1 \mathrm{vvm}$ aeration rate. Bioreactors were inoculated with $1 \mathrm{ml}$ frozen mycelia stock. The bioreactors were equipped with a cooling condenser to avoid evaporation from the medium. $\mathrm{pH}$ was maintained using 2 $\mathrm{M} \mathrm{NaOH}$ solution. The concentrations of $\mathrm{CO}_{2}$ and $\mathrm{O}_{2}$ of the off-gas were monitored using an acoustic gas analyzer (1311, Innova Air Tech Instruments A/S. Naerum, Denmark).

For shake flask cultivations, $50 \mu$ frozen mycelia stock was mixed with $50 \mathrm{ml}$ of sterile medium and added to each flask. Shake flasks were incubated at $28^{\circ} \mathrm{C}$ and 150 rpm. For batch cultivations performed in MTPs, $80 \mu \mathrm{l}$ of frozen mycelia stock was mixed with $80 \mathrm{ml}$ of sterile re-constituted medium. $3.15 \mathrm{ml}$ of this mixture was dispensed into each well of a sterile MTP. The MTPs were incubated at $28^{\circ} \mathrm{C}$ and $150 \mathrm{rpm}$. The shakers used for 
shake flasks and microtiter plate cultivations were orbital shakers with $25 \mathrm{~mm}$ diameter of shaking.

\section{Sampling}

The samples were taken at regular intervals throughout the cultivations for analyses of $\mathrm{pH}$, glucose and antibiotics. For bioreactor cultivations, $13 \mathrm{ml}$ sample was taken at each time point and $10 \mathrm{ml}$ was used for dry cell measurement $(\mathrm{DCW})$. When $\mathrm{OD}_{450} \mathrm{~nm}$ of biomass was above 1, the quantity of broth for each DCW determination was reduced to $5 \mathrm{ml}$ and duplicate measurements were analyzed. The remaining $3 \mathrm{ml}$ sample was preserved for later antibiotic quantification.

For MTPs optical density was used initially for biomass measurements. When $\mathrm{OD}_{450 \mathrm{~nm}}>1$ samples were taken for dry cell weight (in duplicate). Around $2.8 \mathrm{ml}$ broth from each MTP well was taken and $2.4 \mathrm{ml}$ was used for DCW and the remaining $400 \mu \mathrm{l}$ sample was preserved for antibiotic quantification. Thorough mixing of the sample was ensured while pipetting.

\section{Dry cell weight $(\mathrm{DCW})$ measurement}

Dry cell weights were measured as described by Borodina et al. (2008) [18]. Filtrates were preserved for analysis of extracellular metabolites.

\section{Actinorhodin and Udecylprodigiosin quantification}

To extract actinorhodin (ACT), $1.8 \mathrm{ml}$ of $2 \mathrm{M} \mathrm{NaOH}$ was added to $200 \mu \mathrm{l}$ sample. The mixture was vortexed and centrifuged at $10000 \times \mathrm{g}$ for 10 minutes at $4^{\circ} \mathrm{C}$ and the absorbance of the supernatant measured at $640 \mathrm{~nm}$. For extracting undecylprodigiosin (UDP), $1.8 \mathrm{ml}$ of acidified methanol ( $\mathrm{pH}$ 1.5) was mixed with $200 \mu \mathrm{l}$ sample. The entire mixture was kept on shaking overnight at $2^{\circ}$ C. The absorbance of the supernatant was measured at $530 \mathrm{~nm}$ after centrifugation at $10000 \times \mathrm{g}$ for 10 minutes. The antibiotic concentrations were calculated as described in Borodina et al. (2008) [18]. All the measurements were done in duplicate.

\section{Analysis of substrate and extracellular metabolites}

The concentrations of glucose and organic acids were analyzed by HPLC using a HPX-87H column (Bio-Rad Laboratories, California, USA). The operating temperature was $60^{\circ} \mathrm{C}$. Separation was achieved using $5 \mathrm{mM}$ $\mathrm{H}_{2} \mathrm{SO}_{4}$ at a flow rate of $0.6 \mathrm{ml} / \mathrm{min}$. The HPLC was equipped with a Waters 410 differential refractometer (Millipore) and a tunable absorbance detector set at 210 nm (Waters 486, Millipore, Massachusetts, USA).

\section{Volumetric particle size distribution measurements}

A Mastersizer 2000 (Malvern Instruments Ltd, Worcestershire, UK) unit was used to determine the volumetric cell size distribution in the cultivation broth from MTPs and bench-scale reactors (modified from Petersen et al. 2008 [19]). This is a light scattering method that infers a volumetric size distribution, reporting the sizes of spheres with equal volume to the particles actually present. In the size distribution data the particles are divided into 31 size classes ranging from 4.75 to 1,610 $\mu \mathrm{m}$ in diameter and for each size class the volume percentage of the particles is reported. The unit consists of two parts, a mixing chamber with agitator and a laser obscuration cell. The mixing chamber was thoroughly washed with MQ water prior to measurements. For the particle size distribution analysis, $25 \mathrm{ml}$ of MQ water was added to the mixing chamber and agitator speed was fixed at $1000 \mathrm{rpm}$. The broth sample was added until the laser obscuration value reached $12-15 \%$. The measurement was then started and data was recorded. This procedure was repeated three times using the same sample for calculating the average particle size from MTP and bioreactor cultivations, respectively.

\section{Results}

\section{Optimization of inoculum procedure}

To ensure equal distribution of mycelia between wells in the MTPs and also to avoid a time consuming pre-cultivation procedure, we introduced a "single step inoculation" strategy in the form of frozen mycelia, (Figure 1; see also Materials and Methods for more details on the procedure). The mycelia were gently crushed before freezing to minimize pellet formation and to ensure equal distribution among wells when inoculating MTPs. No significant difference was observed in cultivation trajectories when using FM compared to cultivations using the classical method of inoculation (data not shown; [18]), indicating that the crushing of mycelia had no adverse effects on growth and production. This inoculation procedure using FM was applied for all cultivations described below.

\section{Performance of un-optimized MTP cultivations}

The specific growth rate was $0.03 \pm 0.008 \mathrm{~h}^{-1}$ across all the MTPs, which was less than the reported specific growth rate of $0.1 \mathrm{~h}^{-1}$ for $S$. coelicolor in bench-scale reactors (Table 1) [18]. Around $15 \mathrm{hrs}$ of lag phase was seen in all the cultivations. In terms of antibiotic production, onset of undecylprodigiosin (UDP) was observed around 25 hrs where as actinorhodin (ACT) started at around $50 \mathrm{hrs}$. The final titer (at $100 \mathrm{hrs)} \mathrm{was}$ $6.4 \mathrm{mg} / \mathrm{l}$ and $18.5 \mathrm{mg} / \mathrm{l}$ for UDP and ACT respectively, which was a lot lower than normally seen in bench-scale reactors (Table 1 ).

\section{Optimization of MTP cultivations a) Effect of bead size}

In the search for what may cause the poor behavior of the MTP cultivations, large differences in morphology 
Table 1 Macro parameters in MTP, Shake flask and Bioreactor

\begin{tabular}{lcccc}
\hline & $\begin{array}{c}\text { Un- } \\
\text { optimized } \\
\text { MTP }\end{array}$ & $\begin{array}{c}\text { Optimized } \\
\text { MTP }^{2}\end{array}$ & SF $^{2}$ & Bioreactor $^{3}$ \\
\hline $\boldsymbol{\mu}_{\max }\left(\mathbf{h}^{-1}\right)$ & $0.03 \pm 0.008$ & $0.11 \pm 0.01$ & $0.11 \pm$ & $0.10 \pm$ \\
Final Biomass (g/ & $2.0 \pm 0.13$ & $4.0 \pm 0.13$ & $3.7 \pm 1.3$ & $4.1 \pm 0.1$ \\
L) & & & & \\
UDP (mg/L) & $6.4 \pm 1.6$ & $80 \pm 4.4$ & $28 \pm 15.5$ & $65 \pm 3.3$ \\
ACT $(\mathbf{m g} / \mathbf{L})$ & $18.5 .0 \pm 7.5$ & $520 \pm 17.0$ & $267 \pm$ & $500 \pm 17.7$ \\
& & & 23.6 & \\
\hline
\end{tabular}

Biomass, UDP and ACT concentration indicated in the table corresponds to 120 hrs.

'Data based on 4 biological replicates each in triplicate. Un-optimized MTPs contained a medium with MES buffer and no glass beads.

${ }^{2}$ Data based on 3 biological replicates each in triplicate. Optimized MTPs and Shake flasks (SF) contained a medium with $100 \mathrm{mM}$ MOPS buffer to maintain constant $\mathrm{pH}$ and $3 \mathrm{~mm}$ glass beads.

${ }^{3}$ Data based on three cultivations.

between bench-scale and MTP cultivations were observed. In MTPs, formation of large pellets took place, which was believed to cause well to well variation in terms of growth and antibiotic production and hence, to a large extent be responsible for the poor performance. To avoid pellet formation in MTPs, glass beads were added to each well (six beads per well). Bead sizes in the range 0.75-4.0 $\mathrm{mm}$ were evaluated. Samples were taken at regular intervals to monitor growth, antibiotic production as well as volumetric particle size distribution of the biomass. Pellet formation was observed in the MTPs with no beads as well as with $0.75 \mathrm{~mm}$ zirconium and $2 \mathrm{~mm}$ glass beads. The average size of these pellets was in the range of 255 to $330 \mu \mathrm{m}$ (Figure 2). Dispersed morphology was observed in the MTPs with glass beads of 3 and $4 \mathrm{~mm}$ size. We observed that addition of $3 \mathrm{~mm}$ beads not only helped in maintaining the morphology in a narrow size window of $58 \pm 8 \mu \mathrm{m}$, but also played a significant role towards preventing wall growth. Another major advantage of using glass beads was high reproducibility of mycelial size distribution. The morphology of mycelia not only remained within an acceptable variation limit throughout a cultivation cycle (Figure 3a) but also a high degree of reproducibility was maintained across the wells (Figure $3 \mathrm{~b}$ ). This is very important while screening large numbers of strains or cultivation conditions where morphological reproducibility is one of the key influencing factors. Surprisingly a small peak representing 200 to $400 \mu \mathrm{m}$ mycelia was observed at 96 hrs (Figure 3a). However, no pellets were detected during microscopic examination and the overall $\%$ of this peak is below significance $(\leq 0.8 \%$ of total biomass volume). For the cultivations with $3 \mathrm{~mm}$ beads, ACT and UDP production was also significantly improved, approaching values observed for bench-scale

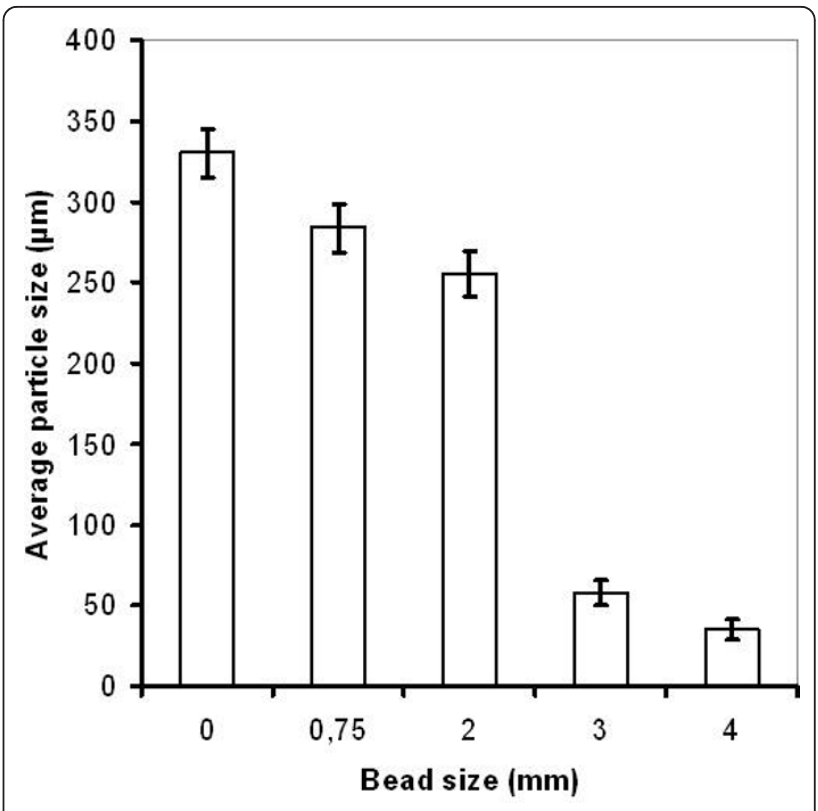

Figure 2 Effect of bead size on S. coelicolor morphology. Each MTP containing beads of one of the following sizes (no beads, 0.75 , 2, 3 and $4 \mathrm{~mm}$ ) was inoculated with S. coelicolor. About $1 \mathrm{ml}$ sample (taken at $48 \mathrm{hrs}$ ) from each MTP was loaded into the mixing chamber of Mastersizer 2000 to determine the size distribution. Data shown are mean values from three wells and error bars represent standard deviation.

reactors (data not shown). The average particle size was slightly reduced $35 \pm 6 \mu \mathrm{m}$ in the cultivations with $4 \mathrm{~mm}$ beads (Figure 2). No ACT production was observed in MTPs with $4 \mathrm{~mm}$ beads up to $100 \mathrm{hrs}$. Hence, we conclude that the use of $3 \mathrm{~mm}$ beads is optimal for S. coelicolor MTP cultivations. Interestingly we observed multiple size distribution in the cultivations conducted in reactors (Figure 3c). The morphology was more pellet-like (200$400 \mu \mathrm{m})$ at the early state of cultivation, i.e. before 40 hrs, whereas a mixed size distribution was observed in the later stages of cultivation where the particles with the same size range as seen for MTPs increased in number. Similar behavior has been reported by Petersen et al. 2011 [20]. They observed a change in morphology during fed-batch cultivations with $S$. coelicolor, with a smaller size population appearing at around $30-50 \mathrm{~h}$ and then increasing in number throughout the cultivation. The shear exerted from agitation might be one of the possible reasons behind the observed morphologies.

\section{b) Effect of concentration of MOPS buffer}

Significant decrease in $\mathrm{pH}$ is another factor that may contribute to the poor behavior observed for the unoptimized MTP cultivations. Hence, the MES buffer was replaced with MOPS, which has a higher $\mathrm{pK}_{\mathrm{a}}$ value (7.20 instead of 6.15) with the ability to keep $\mathrm{pH}$ above 6.5 and possible inhibitory concentrations of MOPS 

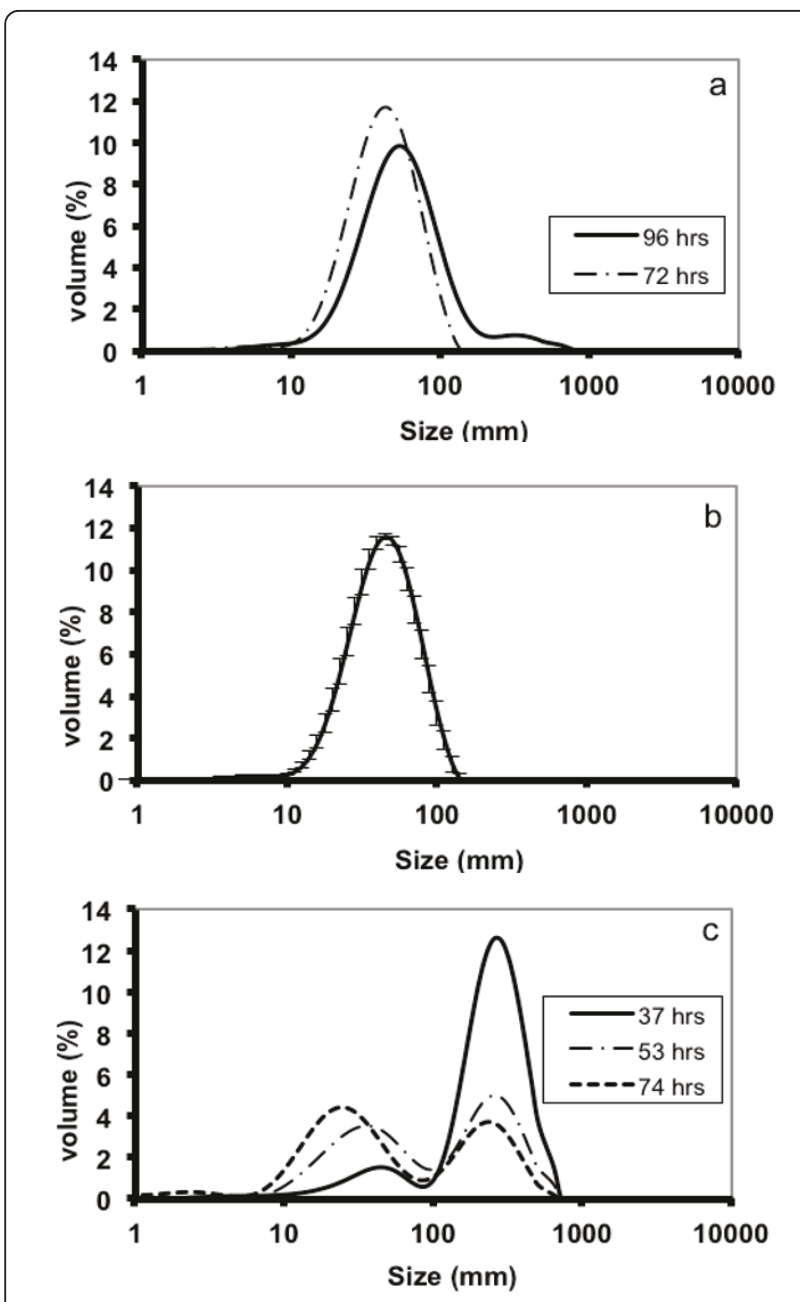

Figure 3 Morphology of S. coelicolor across scales. a) Particle size distributions at two different time points during growth in MTP. Samples were taken at 72 and 96 hrs from three wells and mean values for each time point is reported. b) Reproducibility across wells in MTPs. Samples was taken from three wells at 72 hrs to estimate mean values. (c) Particle size distribution in bioreactor cultivations. Three samples were taken at each time point (37, 53 and $74 \mathrm{hrs})$. Mean values are presented in the figure.

were evaluated by testing different buffer strengths. $S$. coelicolor FM stock was dispensed in defined medium containing 50,100,130, 160 and $190 \mathrm{mM}$ of MOPS, respectively. This pre-inoculated medium was then dispensed in five MTPs (one plate per MOPS concentration) containing six glass beads/well (ø $3 \mathrm{~mm})$. Samples were taken in triplicate at regular intervals to monitor antibiotic production and growth. A significant delay in ACT onset was observed in the batches with higher MOPS concentration (data not shown), which resulted in lower ACT concentrations at the end of the cultivations. ACT concentration was decreased significantly in the batches with 160 and $190 \mathrm{mM}$ MOPS (Figure 4a).
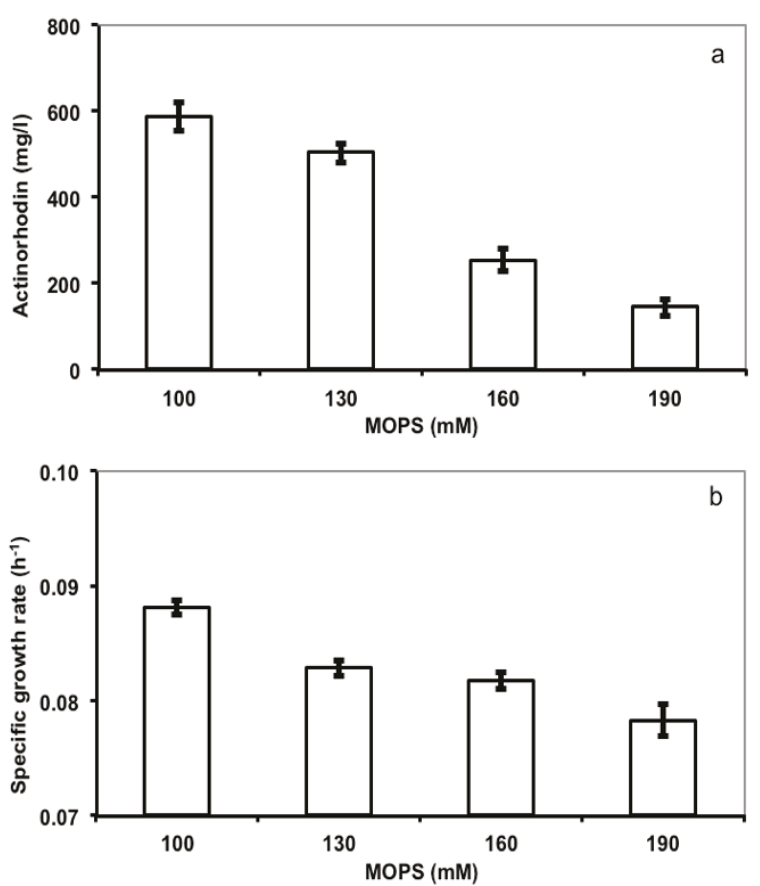

Figure 4 Effect of MOPS buffer on a) Actinorhodin titer b) Specific growth rate. Five different MOPS concentrations (50, 100, 130, 160 and $190 \mathrm{mM}$ ) were investigated. The concentration of actinorhodin described in the figure is from 144 hrs. Samples from 0 to $48 \mathrm{hrs}$ were used to estimate specific growth rate. Data presented are mean values of three samples. Please note that data from cultivations with $50 \mathrm{mM}$ MOPS was not included in the figure as $\mathrm{pH}$ decreased to 5.80 early during cultivation, severely affecting growth and production.

Growth rate did also decline with higher MOPS concentrations, although not as drastically as the ACT production (Figure $4 \mathrm{~b}$ ). In cultivations with $50 \mathrm{mM}$ MOPS, $\mathrm{pH}$ decreased to 5.8 with poor growth and production as a result, demonstrating that the buffering capacity was not enough at this buffer concentration (data not shown). Consequently, MOPS buffer concentration of $100 \mathrm{mM}$ was optimal for cultivations in terms of onset of ACT production, amounts of ACT produced and growth.

\section{Validation and performance of optimized MTP}

Once the MTP platform was optimized, S. coelicolor cultivations were carried out in MTPs, shake flasks and bioreactors (each in triplicate) in order to evaluate the reproducibility of the MTP cultivations and to compare the performance at different scales $(3 \mathrm{ml}, 50 \mathrm{ml}$ and 1 L, respectively). For MTP an additional fourth cultivation was carried out two weeks after the first three, to further check reproducibility. In all cases phosphate limited medium was employed and FM stock was used as inoculum. For shake flasks and MTPs, the medium was 
buffered with $100 \mathrm{mM}$ MOPS, whereas $2 \mathrm{M} \mathrm{NaOH}$ was used to control the $\mathrm{pH}$ at 6.80 in the reactors. Samples were drawn at regular intervals in triplicate. For validating rates of underlying metabolic processes, analysis of physiological traits was performed using standard physiological extracellular parameters.

The mean specific growth rate was $0.11 \mathrm{~h}^{-1}( \pm 0.01)$ across all the MTPs (Table 1, 2). Around $15 \mathrm{~h}$ of lag phase was seen in all the plates (Figure 5). In terms of antibiotic production, onset of UDP was observed around $40 \mathrm{~h}$ where as ACT production started at around $60 \mathrm{~h}$. The titer at $120 \mathrm{~h}$ was $80 \mathrm{mg} / \mathrm{l}$ and 520 $\mathrm{mg} / \mathrm{l}$ for UDP and ACT respectively. The final biomass concentration was $4.0 \mathrm{~g} / \mathrm{L}$. The $\mathrm{pH}$ was typically reduced to 6.5 from 6.9 at $100 \mathrm{~h}$ of cultivation and further to 6.0 at $155 \mathrm{~h}$. It should be noted that in the cultivations with MES buffer (in un-optimized MTPs), $\mathrm{pH}$ dropped to 5.8 immediately after the exponential growth phase at around $40 \mathrm{~h}$. The standard deviation was in the range of $\pm 5-10 \%$ for biomass and antibiotic measurements across all the optimized MTPs. Moreover, physiological parameters were within SD limits of $\pm 10 \%$ (data not shown) for cultivations carried out with different frozen mycelia lots, demonstrating good reproducibility using the optimized MTP procedure.

In bioreactors a lag phase of around 20 hours was observed (Figure 6.). Time points for onset of UDP and ACT production were the same as those for the MTP cultivations, around 40 hours and 60 hours, respectively. A mean specific growth rate of $0.10 \pm 0.01$ was observed and titers of UDP and ACT reached $65 \mathrm{mg} / \mathrm{L}$ and 500 $\mathrm{mg} / \mathrm{L}$, respectively, at $120 \mathrm{hrs}$. The biomass concentration was $4.1 \mathrm{~g} / \mathrm{L}$ at the same time point.

Also the kinetic parameters calculated from data obtained in MTPs and bench-scale cultivations were in agreement (Tables 1, 2). Volumetric antibiotic production rates were found to be around $2 \mathrm{mg} / \mathrm{L} / \mathrm{h}$ and 10

Table 2 Macro parameters in MTPs and in Bioreactors

\begin{tabular}{|c|c|c|c|}
\hline MacroParameters & MTP1 & MTP2 & MTP3 \\
\hline$\mu_{\max }\left(h^{-1}\right)$ & $0.10 \pm 0.002$ & $0.12 \pm 0.01$ & $0.11 \pm 0.001$ \\
\hline q uDP (mg/L/h) & $1.9 \pm 0.09$ & $1.8 \pm 0.13$ & $2.25 \pm 0.2$ \\
\hline $\mathrm{q}_{\mathrm{ACT}}(\mathrm{mg} / \mathrm{L} / \mathrm{h})$ & $10.9 \pm 0.15$ & $8.02 \pm 0.6$ & $9.7 \pm 1.4$ \\
\hline$-r_{s}$ (mmoles glu/g DCW/h) & $1.8 \pm 0.07$ & $1.8 \pm 0.07$ & $1.8 \pm 0.11$ \\
\hline$Y_{5 x}(g / g)$ & $0.32 \pm 0.01$ & $0.32 \pm 0.01$ & $0.35 \pm 0.02$ \\
\hline MacroParameters & $\mathrm{R} 1$ & $\mathrm{R} 2$ & R3 \\
\hline$\mu_{\max }\left(h^{-1}\right)$ & $0.10 \pm 0.01$ & $0.10 \pm 0.001$ & $0.11 \pm 0.002$ \\
\hline $\mathrm{q}$ uDP $(\mathrm{mg} / \mathrm{L} / \mathrm{h})$ & $1.8 \pm 0.02$ & $1.9 \pm 0.13$ & $2.1 \pm 0.12$ \\
\hline$q_{\text {ACT }}(\mathrm{mg} / \mathrm{L} / \mathrm{h})$ & 11.6 & $12.4 \pm 0.31$ & $10.7 \pm 0.001$ \\
\hline$-r_{\mathrm{s}}$ (mmoles glu/g DCW/h) & $1.9 \pm 0.2$ & $1.82 \pm 0.005$ & $1.59 \pm 0.13$ \\
\hline$Y_{5 x}(g / g)$ & $0.36 \pm 0.05$ & $0.31 \pm 0.001$ & $0.31 \pm 0.02$ \\
\hline
\end{tabular}

Triplicate samples were taken from MTPs and Bioreactors at various time points throughout the cultivations to calculate macro parameters indicated in the table. $\mathrm{mg} / \mathrm{L} / \mathrm{h}$ for UDP and ACT respectively, for both MTPs and bioreactors. One could argue that the ACT production was somewhat lower in MTPs as compared to reactors however, the difference was very small. Specific glucose consumption rates $\left(-\mathrm{r}_{\mathrm{s}}\right)$ were in the range of 1.6 to $1.9 \mathrm{mmoles} . g l \mathrm{c} / \mathrm{g} . \mathrm{dcw} / \mathrm{h}$. Biomass yields were estimated to be around 0.33 (g.dcw/g.glucose) across the scales. The overall standard deviation was below $\pm 10 \%$ for all the parameters.

In the overall comparison of the physiological parameters, MTP and bioreactor cultivations showed good agreement. However, shake flask data differed quite a lot (Table 1). Considerably lower antibiotic production was observed and the reproducibility was a lot poorer than for MTPs and bioreactors. This was likely due to variations in morphology between flasks and problems with pellet formation and wall growth.

\section{Discussion}

Discovery of new antibiotics and improvement of existing strains and processes are major goals for the fermentation industry. Efficient discovery and improvement programs require high-throughput cultivations platforms. The outcome of these screenings is highly dependent on high reproducibility. Furthermore, well-controlled conditions are essential to allow for extrapolation of the results to larger scales. The current study demonstrates the successful development of a deepwell MTP-based platform for cultivation of $S$. coelicolor and investigations of secondary metabolite production. Optimization of the inoculation procedure, the use of crushed, frozen mycelia, optimization of the buffer and addition of glass beads resulted in high reproducibility and that the same performance as in bench-scale reactors could be achieved (STDDEV: 5-10\%). Hence, the 24-square deepwell MTP platform described here provides a reliable cultivation platform for understanding physiology and metabolic behavior. The MTP method that we developed has been successfully used by Siebenberg et al. (2010) and Dangel et al. (2010) [16,21] to investigate production of novobiocin. Moreover, the platform has also in our lab been effectively applied to study secondary metabolite production by other actinomycetes with only minor adjustments, e.g. glycopeptides production by Amycolatopsis balhimycina [unpublished data].

At the beginning of this work, before optimization, we observed significant discrepancy with respect to specific growth rate and volumetric production rate as compared to bioreactors and shake flasks, which is in agreement with the report by Minas et al. (2000) [22]. Well to well variation was another observation. This discrepancy was most likely a result of the pellety morphology in MTPs. This was addressed during the optimization procedure by inclusion of MOPS buffer and addition of 


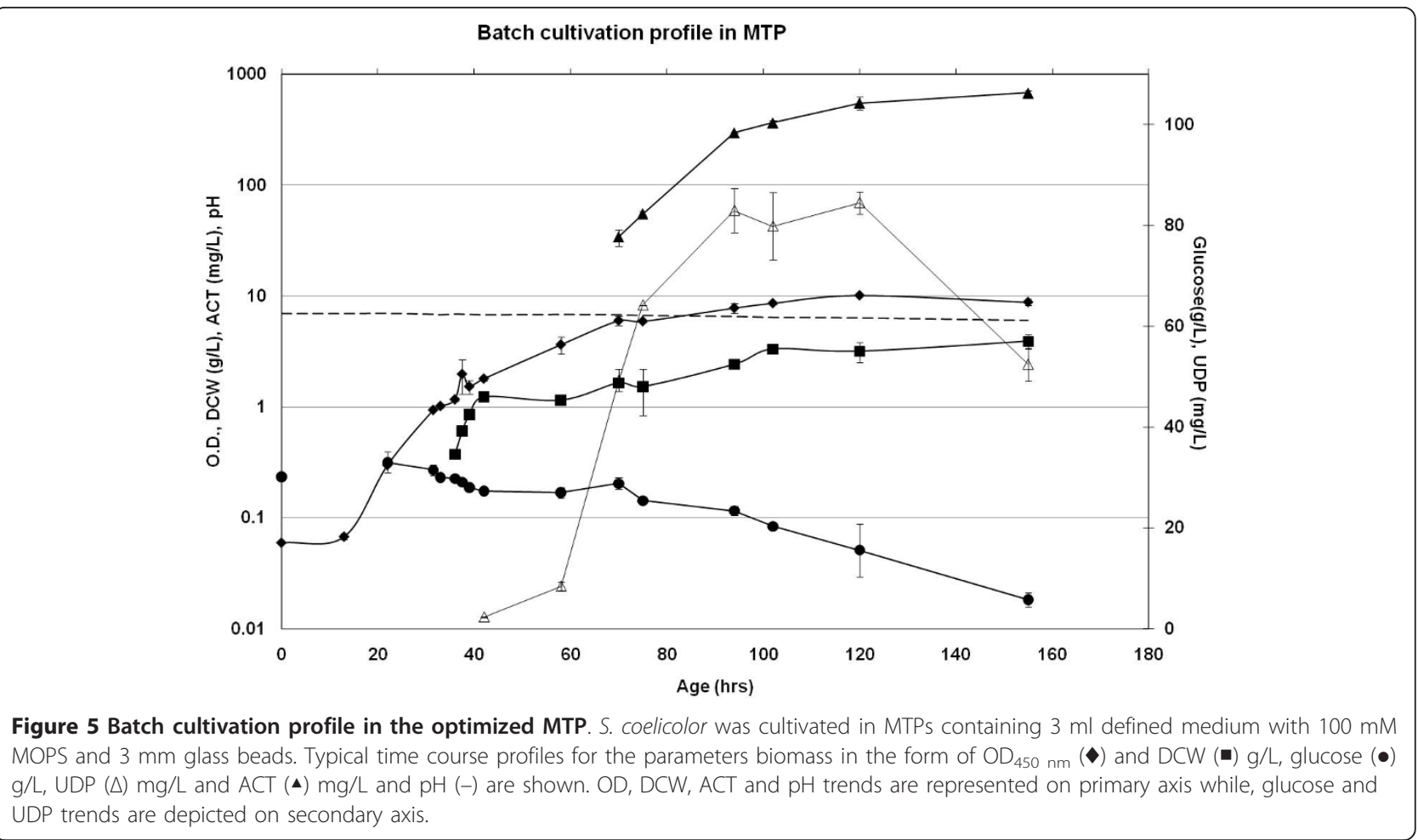

glass beads, resulting in a dispersed, highly reproducible morphology and physiological behavior.

Classical methods of propagation include preparation of spore plates, harvest of spores and inoculation of spores into seed medium for germination. The resulting culture is then inoculated into production medium. This procedure might introduce variability at each step and is rather time consuming. An alternative could be to

\section{Batch cultivation profile in reactor}

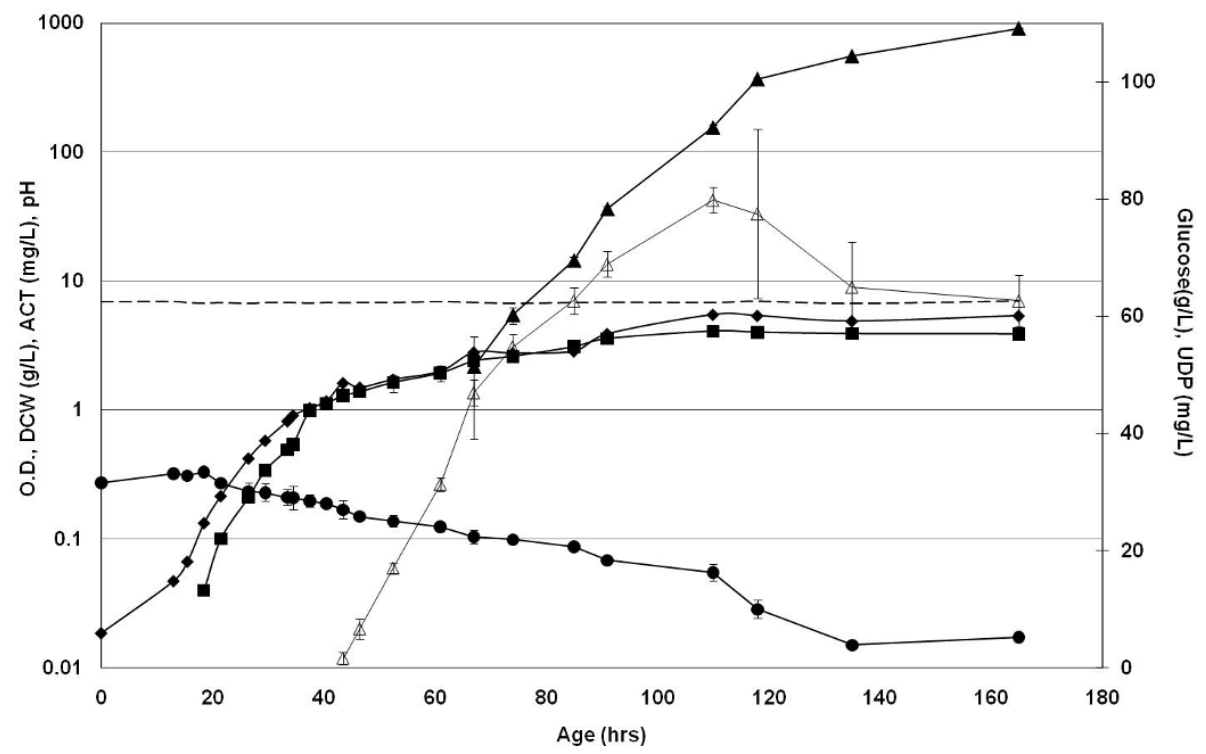

Figure 6 Batch cultivation profile in the reactor. S. coelicolor was cultivated in a reactor with $1 \mathrm{~L}$ defined medium. Typical time course profiles for the parameters biomass in the form of $\mathrm{OD}_{450 \mathrm{~nm}}(\bullet)$ and DCW $(\bullet) \mathrm{g} / \mathrm{L}$, glucose $(\bullet) \mathrm{g} / \mathrm{L}, \mathrm{UDP}(\Delta) \mathrm{mg} / \mathrm{L}, \mathrm{ACT}(\boldsymbol{\Delta}) \mathrm{mg} / \mathrm{L}$ and $\mathrm{pH}(-)$ are shown. OD, DCW, ACT and pH trends are represented on primary axis while, glucose and UDP trends are depicted on secondary axis. 
directly inoculate with spores in the production medium. However, spores are hydrophobic in nature and hence equal distribution in the culture medium may be problematic. Also, direct inoculation of spores into production medium may lead to longer lag phase, particularly if a minimal medium is used in this step. To circumvent these drawbacks, we applied frozen mycelia for direct inoculation, which we earlier successfully have applied to novobiocin production [16]. Both the classical method, with a pre-germination step, and the procedure with direct inoculation of spores require preparation of fresh spores and that takes approximately 8 days. A main advantage of using FM is that the time required to start the main cultivation is drastically reduced. In addition, the dispersed property enhances equal distribution of cells, making FM convenient to use for inoculation of miniaturized versions of cultivations and improves reproducibility.

Shake flasks have traditionally been the choice for screening studies as they allow for relatively small volumes and are easy to set up and clean compared to bench-scale reactors. The drawback compared to benchscale is typically that conditions are less controlled, limitations in mass transfer may be an issue and the limitation in volume often results in that only end-point samples are taken. We observed high variations between shake flasks cultivations and reduced final concentrations and volumetric production rates for antibiotics. Our results are in agreement with those obtained by Siebenberg et al. (2010) [16] that also report lower final antibiotic concentrations and higher variation in data for shake flasks compared to MTPs. There are studies demonstrating superior oxygen transfer rates in deepwell MTPs compared to shake flasks [15] and we believe that this in addition to difficulties with controlling the morphology may be important underlying reasons for the poor performance of shake flasks as antibiotic production is known to be greatly influenced by oxygen levels and morphology.

\section{Conclusion}

24-square deepwell plates with $3 \mathrm{ml}$ working volume were used for S. coelicolor A3(2) cultivations. 6 beads ( $\varnothing$ $3 \mathrm{~mm}$ ) were included in each well and MOPS buffer was added to the medium to achieve dispersed and reproducible morphology. A comparative study was undertaken to verify agreement of extracellular parameters (specific growth rate, volumetric production rates for antibiotics) across different scales i.e. bioreactors $(1 \mathrm{~L})$, shake flasks $(50 \mathrm{ml})$ and MTPs $(3 \mathrm{ml})$. The MTP platform developed here performed highly reproducible and physiological data demonstrated good agreement with data from bioreactors. Shake flasks showed discrepancy with respect to significantly reduced volumetric production rates of antibiotics. Hence, MTPs are preferred over shake flasks for high-throughput physiology studies in filamentous microorganisms.

\section{Abbreviations}

exp: Exponential; UDP: Undecylprodigiocin; ACT: Actinorhodin; $\mu_{\max , \text { : Maximal }}$ specific growth rate; MTPs: Microtiter plates; SFs: Shake flasks; DCW: Dry cell weight; FM: Frozen mycelia; q: Volumetric production rate; $Y_{\text {sx }}$ : Biomass yield on substrate; $r_{s}$ : Specific glucose consumption rate.

\section{Acknowledgements}

We would like to thank the Technical University of Denmark, the Danish biotechnology research school and the European Commission (IP005224 ActinoGEN) for financial support during this work. We would like to acknowledge Prof. Jens Nielsen for initial discussions on scale down and optimization of microtiter plates.

\section{Author details}

${ }^{1}$ Center for Microbial Biotechnology, Department of Systems Biology, Technical University of Denmark, Building 223, DK-2800 Kgs Lyngby, Denmark. ${ }^{2}$ Present address: Novozymes A/S, Krogshoejvej 36, 2880 Bagsvaerd, Denmark. ${ }^{3}$ Present address: Novozymes A/S, Hallas Alle 1, 4400 Kalundborg, Denmark.

\section{Authors' contributions}

SVS and PMB carried out the experimental work, performed data analysis and contributed to drafting of the manuscript. AEL initiated the study and contributed to experimental design, interpretation of data and manuscript writing. All authors read and approved the final manuscript.

\section{Competing interests}

The authors declare that they have no competing interests.

Received: 30 August 2011 Accepted: 17 January 2012

Published: 17 January 2012

\section{References}

1. Challis GL, Hopwood DA: Synergy and contingency as driving forces for the evolution of multiple secondary metabolite production by Streptomyces species. Proc Natl Acad Sci USA 2003, 100:14555-14561.

2. Kisser T, Bibb MJ, Buttner MJ, Chater KF, Hopwood DA: Practical Streptomyces Genetics. Norwich, England: The John Innes Foundation; 2000.

3. Baltz RH: Genetic methods and strategies for secondary metabolite yield improvement in actinomycetes. Antonie Van Leeuwenhoek International Journal of General and Molecular Microbiology 2001, 79(3-4):251-259.

4. Rokem JS, Lantz AE, Nielsen J: Systems biology of antibiotic production by microorganisms. Nat Prod Rep 2007, 24(6):1262-1287.

5. Patil KR, Akesson M, Nielsen J: Use of genome-scale microbial models for metabolic engineering. Curr Opin Biotechnol 2004, 15(1):64-69.

6. Koffas M, Roberge C, Lee K, Stephanopoulos G: Metabolic engineering. Annu Rev Biomed Eng 1999, 1:535-557.

7. Shuler MJ, Kargi F: Bioprocess Engineering: Basic Concepts. Prentice Hall; 22001.

8. Kumar S, Wittmann C, Heinzle E: Minibioreactors. Biotechnol Lett 2004, 26(1):1-10

9. Micheletti M, Lye GJ: Microscale bioprocess optimisation. Curr Opin Biotechnol 2006, 17(6):611-618.

10. Kitaoka M, Robyt JF: Use of a microtiter plate screening method for obtaining Leuconostoc mesenteroides mutants constitutive for glucansucrase. Enzyme Microb Technol 1998, 22(6):527-531.

11. Fischer E, Sauer U: Metabolic flux profiling of Escherichia coli mutants in central carbon metabolism using GC-MS. European Journal of Biochemistry 2003, 270(5):880-891.

12. Betts Jl, Baganz F: Miniature bioreactors: current practices and future opportunities. Microbial Cell Factories 2006, 5:21.

13. Duetz WA: Microtiter plates as mini-bioreactors: miniaturization of fermentation methods. Trends Microbio/ 2007, 15(10):469-475. 
14. Islam RS, Tisi D, Levy MS, Lye GJ: Framework for the rapid optimization of soluble protein expression in Escherichia coli combining microscale experiments and statistical experimental design. Biotechnol Prog 2007, 23(4):785-793.

15. Duetz WA, Witholt B: Oxygen transfer by orbital shaking of square vessels and deepwell microtiter plates of various dimensions. Biochem Eng J 2004, 17(3):181-185.

16. Siebenberg S, Bapat PM, Lantz AE, Gust B, Heide L: Reducing the variability of antibiotic production in Streptomyces by cultivation in 24-square deepwell plates. J Biosci Bioeng 2010, 109(3):230-234.

17. Bapat PM, Bhartiya S, Venkatesh KV, Wangikar PP: Structured kinetic model to represent the utilization of multiple substrates in complex media during rifamycin B fermentation. Biotechnol Bioeng 2006, 93(4):779-790.

18. Borodina I, Siebring J, Zhang J, Smith CP, van Keulen G, Dijkhuizen L, Nielsen J: Antibiotic overproduction in Streptomyces coelicolor A3(2) mediated by phosphofructokinase deletion. J Biol Chem 2008, 283(37):25186-25199.

19. Petersen N, Stocks S, Gernaey KV: Multivariate models for prediction of rheological characteristics of filamentous fermentation broth from the size distribution. Biotechnol Bioeng 2008, 100(1):61-71.

20. Petersen NR, Stocks SM, Eliasson AL, Gernaey KV: Introducing process analytical technology (PAT) in filamentous cultivation process development: comparison of advanced online sensors for biomass measurement. J Industrial Microbiol Biotechnol 2011, 38:1679-1690.

21. Dangel V, Westrich L, Smith MCM, Heide L, Gust B: Use of an inducible promoter for antibiotic productionin a heterologous host. Appl Microbiol Biotechnol 2010, 87(1):261-269.

22. Minas W, Bailey JE, Duetz W: Streptomycetes in micro-cultures: Growth, production of secondary metabolites, and storage and retrieval in the 96-well format. Antonie Van Leeuwenhoek International Journal of General and Molecular Microbiology 2000, 78(3-4):297-305.

doi:10.1186/1475-2859-11-9

Cite this article as: Sohoni et al:: Robust, small-scale cultivation platform for Streptomyces coelicolor. Microbial Cell Factories 2012 11:9.

\section{Submit your next manuscript to BioMed Central and take full advantage of:}

- Convenient online submission

- Thorough peer review

- No space constraints or color figure charges

- Immediate publication on acceptance

- Inclusion in PubMed, CAS, Scopus and Google Scholar

- Research which is freely available for redistribution

Submit your manuscript at www.biomedcentral.com/submit
Ciomed Central 